\title{
Modal liquid crystal microaxicon array
}

\author{
José Francisco Algorri, Virginia Urruchi, Noureddine Bennis, and José Manuel Sánchez-Pena
}

\begin{abstract}
A novel tunable liquid crystal microaxicon array is proposed and experimentally demonstrated. The proposed structure is capable of generating tunable axicons (thousands of elements) of micrometric size, with simple control (four control voltages) and low voltage, and is totally reconfigurable. Depending on the applied voltages, control over the diameter, as well as the effective wedge angle, can be achieved. Controls over the diameter ranging from 107 to $77 \mu \mathrm{m}$ have been demonstrated. In addition, a control over the phase profile tunability, from $12 \pi$ to $24 \pi$ radians, has been demonstrated. This result modifies the effective cone angle. The diameter tunability, as well the effective cone angle, results in a control over the nondiffractive Bessel beam distance. The RMS wavefront deviation from the ideal axicon is only $\lambda / 3$. The proposed device has several advantages over the existing microaxicon arrays, including being simple having a low cost. The device could contribute to developing new applications and to reducing the fabrication costs of current devices.
\end{abstract}

The use of axicons has attracted considerable interest in the last few years because of their wide range of applications in different fields. In contrast with classical lenses, where the shape is usually spherical or cylindrical, this type of optical element is based on a cone shape. This causes a field distribution that is proportional to the zero-order Bessel function $\mathrm{J}_{0}$, also known as nondiffracting Bessel beam [1]. Some properties are selfreconstruction [2] and the appearance of longitudinal components of the field vectors [3]. In the near field, these beams are characterized by a high intensity spot surrounded by rings with a decreasing intensity. The applications are varied and numerous, for example, precision alignment for large telescopes [4] , laser machining [5], medical applications [6,7], commercial applications [8] , optical tweezers [9], and atom optics [10].

Yet, one of the impediments for the broad use of these optical elements is the fabrication cost, usually based on processes that use diamond turning from solid materials such as BK7 glass or fused silica [11]. Some works have proposed the use of liquid crystals (LC). For example, the generation of axicons of several millimeters, based on modal control technique, has been demonstrated [12]. In addition, a structure based on six electrodes and a modal control technique has been proposed to generate an array of four optical elements that can also produce four axicons of several millimeters [13]. Nevertheless, axicons at the micrometric scale, based on this technique, have not been demonstrated until now to the best of our knowledge.

The use of microaxicons in arrays also has important applications such as ultrafast optical communications [14], generation of femtosecond Bessel beams [15], nondiffracting "flying images" [16], and high-resolution optical coherence tomography [17]. Despite this, few works in the literature have demonstrated the fabrication of microaxicon arrays. Some of them use direct electron beam [9], laser direct writing using two-photon polymerization [18], thin-film deposition methods [19], or masks based on the LC displays [20]; these methods tend to have limitations on the axicon profile height and thus the axicon angle. To overcome these drawbacks, another technique based on using a multistep micromolding process [21] has been recently proposed. In general, the proposed ideas use complex fabricating processes. Moreover, a reconfiguration of the final device is not possible.

The parameters defining an axicon are the aperture diameter $(d)$ and the wedge angle $(\gamma)$. In order to increase the nondiffractive distance of a Bessel beam $\left(z_{\max }\right)$, one of these parameters has to be modified $\left(z_{\max } \sim d / \gamma\right)$. Until now, these parameters were modified in the fabrication process. As commented above, the fabrication processes proposed until now produce limitations in the microaxicon parameters. One solution is the use of liquid-crystal-on-silicon spatial light modulators (LCoSSLMs) [22]. Despite this, the main problem is the low phase shift achieved by this device (usually $2 \pi$ ) and the price. Another drawback could be the characteristic structure, based on pixels surrounded by opaque areas, causing losses of light efficiency and possible aliasing in the case of micro-optical systems. To solve this problem some manufacturers use a special anti-reflection coating to ensure that the influence of the pixellated structure is minimized and thus the diffraction losses. In addition, LCoS-SLMs have residual phase distortions and a limited resolution [22]. Another option has been reported [23]. In this work a tunable MEMS axicon mirror array is proposed. It shows a high tunability by controlling the wedge angle with temperature changes. The required temperature control could complicate the final device.

In this Letter, a microaxicon array with high tunability and simple control is proposed. A control over the aperture and effective wedge angle is experimentally demonstrated. This device is simple and reconfigurable with low voltage signals. The proposed structure is capable of generating tunable axicons (thousands of elements) of micrometric size, with simple control (four control voltages) and low voltage, and is totally reconfigurable. The near field measurements demonstrate the generation of a nondiffracting Bessel beam array. Moreover, this 
array is tunable, both in phase amplitude and focus position. This device could contribute to developing new applications and to reducing the fabrication costs of the current devices.

The proposed device comprises two indium-tin oxide ITO coated substrates. The ground and upper planes consist of a pair of comb-type finger electrodes. The substrates are arranged so that their electrodes are in orthogonal position with respect to each other. Over these electrodes a low conductive and transparent polymer layer of poly-(3,4-ethylenedioxythiophene):poly (styrenesulfonic acid) (PEDOT:PSS), is deposited. It is difficult to obtain homogeneous layers with this material. The deposition process has been optimized. The measured square resistance ranges from 7 to $14 \mathrm{M} \Omega / \mathrm{sq}$ for spinner speeds of 3000 and $4500 \mathrm{rpm}$, respectively (30 s). For the latter speed, a better homogeneity has been observed. After a curing time $\left(30 \mathrm{~min}\right.$ at $\left.120^{\circ} \mathrm{C}\right)$, a polyimide layer is deposited by a similar process. This layer is rubbed in order to obtain a molecular homogeneous alignment. The main aligning mechanism is based on the shear stress induced on the alignment layer and the intermolecular interactions between the LC and polyimide. The substrates are separated by spacers of $40 \mu \mathrm{m}$. The resulting cavity is filled with a nematic LC; MDA-981602 from Merck was selected because of its high birefringence, $\Delta n=0.2666$. Figure $\underline{1(a)}$ shows a scheme of the structure comprising these layers. A top view of the electrode pattern is shown in Fig. 1(b). When vertical electrodes are placed perpendicular to the horizontal ones, the confined volume is a cuboid. The top view of this volume is a square area $(107 \mu \mathrm{m} \times 107 \mu \mathrm{m})$. Each comb-type electrode has 51 fingers, resulting in $100 \times 100$

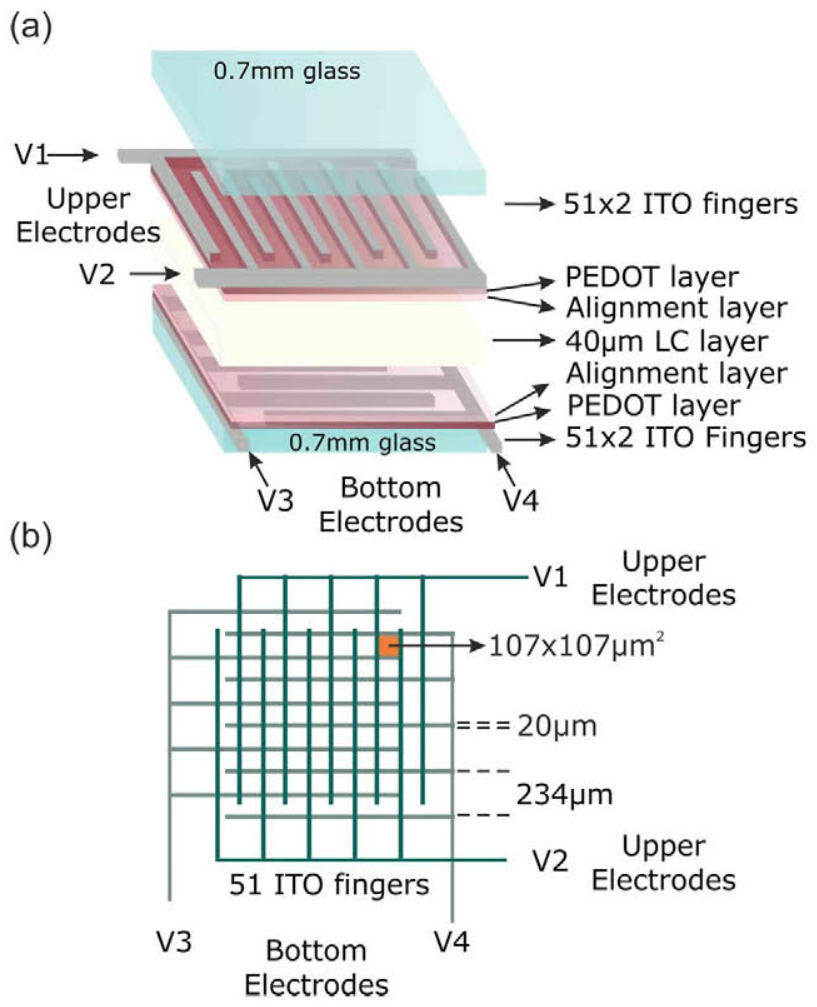

Fig. 1. Tunable microaxicon array. (a) Scheme of the device arrangement and (b) top view of the patterned electrodes. Note: drawings are not to scale. optical elements. Every side of the active area is governed by one different voltage.

The operating principle is based on three physical effects. The first effect is produced by a low resistance between electrodes (in comparison with the LC impedance). This resistance generates a linear voltage distribution from one electrode to another for electrodes on the same side.

The second effect is based on the combination of different electrical phase shifts. When the upper comb-type finger electrodes $\left(V_{1}\right.$ and $\left.V_{2}\right)$ have an electrical phase shift of $180^{\circ}$, between them (e.g., $V_{2}=180^{\circ}$ ), the signals are cancelled at the center of the electrodes. In complex notation, the resulting voltage distribution in the upper side has the shape of a ramp, from $V_{1}$ to $-V_{2}$. The absolute value is a vertical prism array distribution. The same operating principle is required for the bottom electrodes $\left(V_{3}\right.$ and $\left.V_{4}\right)$. In addition, these electrodes have an electrical phase shift of $90^{\circ}$ with respect to the upper electrodes. In complex notation, the resulting voltage distribution is also a ramp, but in the imaginary axis (from $\mathrm{V}_{1} \cdot \mathrm{i}$ to $-\mathrm{V}_{2} \cdot \mathrm{i}$ ).

The third physical effect is related to the nematic LC physics. The molecules of the nematic LC align parallel to the electrical field. This produces an electrically controlled birefringence. In nematic LC the birefringence has a nonlinear relation with the root mean square (RMS) value of the applied voltage. In the proposed device, this voltage is the difference between the upper voltage distribution and the bottom one. Considering the complex notation, and $\mathrm{V}_{1}=\mathrm{V}_{2}=\mathrm{V}_{3}=\mathrm{V}_{4}=\mathrm{V}$, it is easily observed that the corners of the active area have the same absolute value of $\sqrt{ } 2 \cdot \mathrm{V}$. However, the electrical signals in the center of the active area are all cancelled. The result is a voltage distribution with a conical shape. Finally, the molecules of the LC layer are distributed in the same way, producing an optical phase shift with the shape of an axicon.

For the experimental setup, the interference fringes were captured placing test samples between two crossed polarizers with the rubbing direction of the lenticular lenses at $45^{\circ}$ from the linear polarization at the input [Fig. 2(a)]. In order to generate four complex electrical signals, a driving module has been designed and built. It consists of a custom electrical phase shift waveform generator that uses an NI sbRIO-9633 module. This module is an embedded control and acquisition device that integrates a real-time processor and a Xilinx Spartan-6 LX25 FPGA with four 12-bit analog output channels

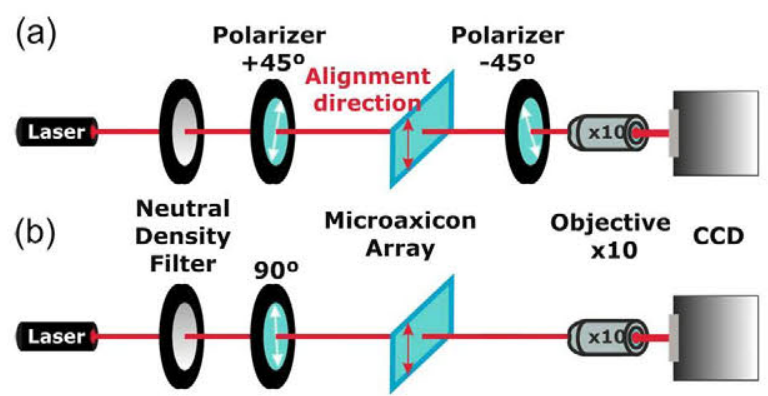

Fig. 2. Experimental setup for (a) interference pattern and (b) intensity measurements. 
$( \pm 10 \mathrm{~V})$. A polarized He-Ne laser beam $(\lambda=632.8 \mathrm{~nm})$ was used as a light source. $A \times 10$ objective was placed in the path of the beam. Finally, interference fringes were recorded by a CCD camera.

Bessel beams were captured by placing test devices behind a parallel polarizer with the rubbing direction of the microaxicon array aligned to the main axis of the polarizer [Fig. 2(b)]. As an example, in Fig. 3 , an interference pattern of two microaxicons and the resulting Bessel beams are shown. The electrical phase shift between electrodes is always maintained between experiments: $\mathrm{V}_{1}=0^{\circ}, \mathrm{V}_{2}=180^{\circ}, \mathrm{V}_{3}=90^{\circ}, \mathrm{V}_{4}=270^{\circ}$. The only modifiable parameter is the RMS amplitude, which is the same at each electrode, $\mathrm{V}_{1}=\mathrm{V}_{2}=\mathrm{V}_{3}=\mathrm{V}_{4}=\mathrm{V}$.

An interesting effect has been observed when the voltage is increased over $4 \mathrm{~V}_{\text {rms }}$. As can be observed in Fig. 3(a), the diameter is smaller than $107 \mu \mathrm{m}$. A clear control over the microaxicon diameter $(d)$, by means of the applied voltage, is demonstrated in Fig. $\underline{4}$. As commented above, this can be used to control the nondiffractive distance of a Bessel beam. Because of the waveform generator limitation, the maximum applied voltage is $10 \mathrm{~V}_{\mathrm{rms}}$. Above $4 \mathrm{~V}_{\mathrm{rms}}$, the microaxicon diameter suffers an approximated reduction of $5 \mu \mathrm{m}$ per $\mathrm{V}_{\mathrm{rms}}$ applied. The diameters range from 107 to $77 \mu \mathrm{m}$ in the voltage range from $4 \mathrm{~V}_{\mathrm{rms}}$ to $10 \mathrm{~V}_{\mathrm{rms}}$. Despite this, the diameter probably decreases linearly if more voltage is applied. The phase shift change is very small in comparison with the diameter reduction. Considering that $z_{\max }$ is modified, when the diameter of the microaxicon is changed, this effect produces a Bessel beam control in another way. One drawback could be an increase of the background signal. The advantages of using this effect depend on the application and need to be further investigated.

Another interesting property of the proposed device is the tunable optical phase of each individual microaxicon, for voltages lower than $4 \mathrm{~V}_{\text {rms }}$. This property modifies the effective wedge angle $(\gamma)$ of the microaxicon that also controls the resulting Bessel beam. The phase profiles were obtained from interference patterns by developing

(a)

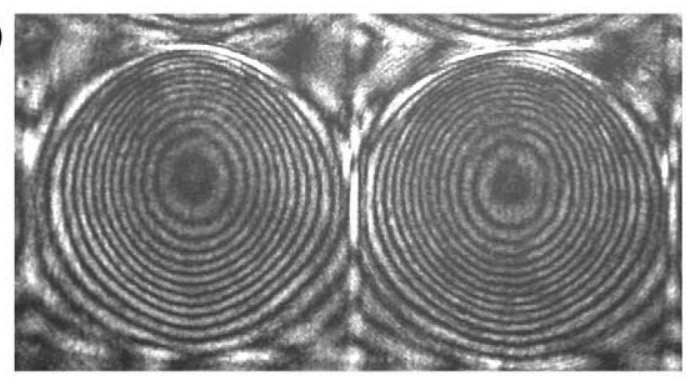

(b)

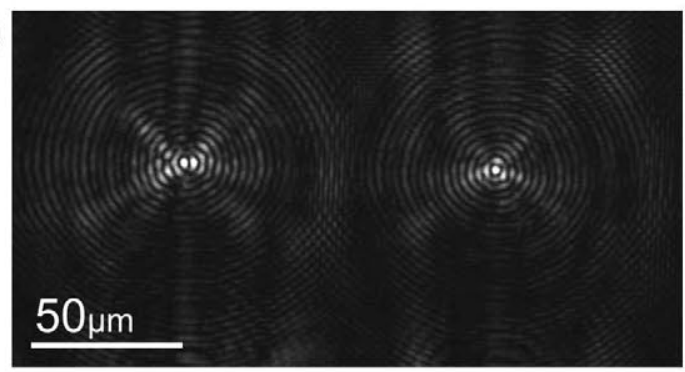

Fig. 3. (a) Interference pattern and (b) intensity distribution of the Bessel beams, for $\mathrm{V}=5 \mathrm{~V}_{\mathrm{rms}}$ and $z=2 \mathrm{~mm}$. (a)

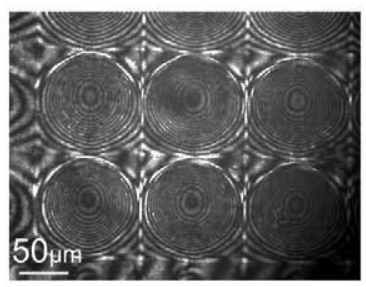

(b)

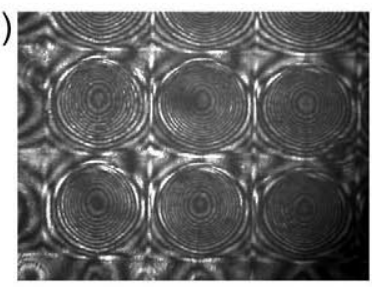

(c)

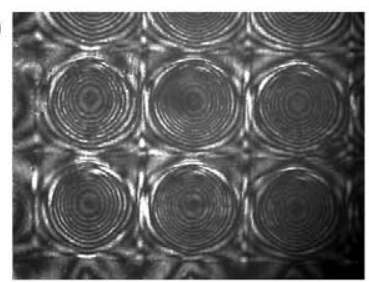

(d)

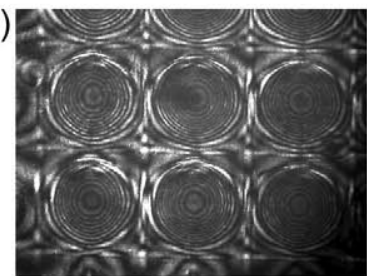

Fig. 4. Tunable diameter for (a) $\mathrm{V}=5 \mathrm{~V}_{\mathrm{rms}}$; (b) $\mathrm{V}=6 \mathrm{~V}_{\mathrm{rms}}$; (c) $\mathrm{V}=7 \mathrm{~V}_{\mathrm{rms}}$; and (d) $\mathrm{V}=8 \mathrm{~V}_{\mathrm{rms}}$.

an image recognition program that processes images and plots optical phase retardation versus horizontal position across the LC microaxicon. The results are shown in Fig. $\underline{5}$. Phase tunability from $12 \pi$ to $24 \pi$ radians is obtained for voltages from $2 \mathrm{~V}_{\mathrm{rms}}$ to $4 \mathrm{~V}_{\mathrm{rms}}$, respectively. As can be seen in Fig. 5 , there is a nonlinear relation between the applied voltage and the maximal phase shift. The measured RMS wavefront error from the ideal axicon is approximately $\lambda / 3$ for the three cases.

In summary, we have described a novel tunable LC microaxicon array and presented the experimental results of its electro-optic behavior. Depending on the applied voltages, a control over the diameter as well as the effective wedge angle can be achieved. Controls over the diameter ranging from 107 to $77 \mu \mathrm{m}$ have been demonstrated. These results are limited by the voltage source output, but everything indicates that higher reductions on the diameter can be possible for higher voltages. In addition, a control over the phase profile, from $12 \pi$ to $24 \pi$ radians, has been also demonstrated. The RMS wavefront deviation from the ideal axicon is only $\lambda / 3$. The realization of a voltage source, which can provide an offset voltage, could reduce the round tip of the microaxicon profile and the RMS wavefront error. Also the

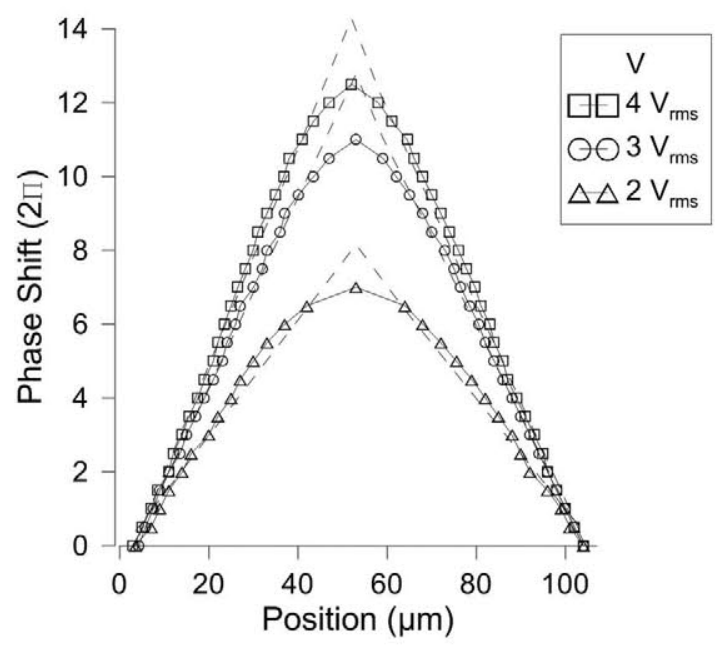

Fig. 5. Tunable phase profile. The continuous lines are the experimental phase profiles. The dashed lines are the ideal axicon profiles. 
inclusion of other types of materials, with high resistivity layer, could improve the microaxicon homogeneity. The reduction of the LC layer could reduce distortions that limit their use in the generation of ultrashort optical needle beams. One solution is a thickness reduction in the LC layer. The same phase shift can be achieved for a higher birefringence LC. These ideas need to be further investigated. The proposed device has several advantages over the existing microaxicon arrays, including simple control and low cost.

This work was supported by the Ministerio de Ciencia e Innovación of Spain (grant no. TEC2009-13991-C02-01) and Comunidad de Madrid (grant no. FACTOTEM2 S2009/ESP-1781).

\section{References}

1. J. Durnin, J. J. Miceli, and J. H. Eberly, Phys. Rev. Lett. 58, 1499 (1987)

2. S. R. Michra, Opt. Commun. 85, 159 (1991).

3. M. Piché, Ch. Varin, and N. McCarthy, Proc. SPIE 3611, 332 (1999).

4. J. H. Mcleod, J. Opt. Soc. Am. 50, 166 (1960).

5. M. Rioux, R. Tremblay, and P. A. Belanguer, Appl. Opt. 17, 1532 (1978).

6. Q. Ren and R. Birngruber, IEEE J. Quantum Electron. 26, 2305 (1990).

7. R. A. Leitgeb, M. Villiger, A. H. Bachmann, L. Steinmann, and T. Lasser, Opt. Lett. 31, 2450 (2006).
8. R. Arimoto, C. Saloma, T. Tanaka, and S. Kawata, Appl. Opt. 31, 6653 (1992).

9. W. C. Cheong, B. P. S. Ahluwalia, X.-C. Yuan, L.-S. Zhang, H. Wang, H. B. Niu, and X. Peng, Appl. Phys. Lett. 87, 024104 (2005)

10. J. Arlt, T. Hitomi, and K. Dholakia, Appl. Phys. B 71, 549 (2000).

11. K. Gourley, I. Golub, and B. Chebbi, Proc. SPIE 7099, $70990 \mathrm{D}$ (2008).

12. A. Kirby, P. Hands, and G. Love, Opt. Express 15, 13496 (2007).

13. J. Algorri, G. Love, and V. Urruchi, Opt. Express 21, 24809 (2013).

14. R. Grumwald and M. Bock, J. Eur. Opt. Soc. Rap. Public. 7, 12009 (2012).

15. R. Grunwald, U. Griebner, F. Tschirschwitz, E. Nibbering, T. Elsaesser, V. Kebbel, H. Hartmann, and W. Jüptner, Opt. Lett. 25, 981 (2000).

16. M. Bock, S. Das, and R. Grunwald, Opt. Express 17, 7465 (2009).

17. K.-S. Lee and J. P. Rolland, Opt. Lett. 33, 1696 (2008).

18. X.-F. Lin, Q.-D. Chen, L.-G. Niu, T. Jiang, W.-Q. Wang, and H.-B. Sun, J. Lightwave Technol. 28, 1256 (2010).

19. R. Grunwald, S. Woggon, R. Ehlert, and W. Reinecke, Pure Appl. Opt. 6, 663 (1997).

20. Q. Peng, Y. Guo, B. Chen, J. Du, J. Xiang, and Z. Cui, Proc. SPIE 4755, 748 (2002).

21. N. Weber, D. Spether, A. Seifert, and H. Zappe, J. Opt. Soc. Am. A 29, 808 (2012).

22. M. Bock, S. Das, and R. Grunwald, Opt. Express 17, 7465 (2009).

23. J. Brunne and U. Wallrabe, Opt. Lett. 38, 1939 (2013). 